\title{
Long-term Follow-up of Percutaneous Vertebroplasty in Osteoporotic Compression Fracture: Minimum of 5 Years Follow-up
}

\author{
Jin Hwan Kim, Si Hoon Yoo, Jung Hoon Kim
}

Department of Orthopedic Surgery, Inje University Ilsan Paik Hospital, Inje University College of Medicine, Goyang, Korea

\begin{abstract}
Study Design: This was designed as a retrospective study.
Purpose: We assessed the radiographic and clinical outcome of patients who underwent percutaneous vertebroplasty (PVP) in osteoporotic compression fractures with a minimum of 5 years follow-up.

Overview of Literature: Percutaneous vertebroplasty is effective surgical method for treating osteoporotic compression fracture.

Methods: Between January 2000 and August 2005, 159 patients were treated with PVP for osteoporotic compression fracture at our department; 43 patients died during follow-up, and 69 patients (121 vertebras) were available for follow-up for over 5 years. We analyzed the clinical and radiologic outcome including cement feature.

Results: The mean follow-up period was 5.7 years. Clinical outcome by mean visual analogue scale (VAS) score revealed a decreased 4.9 points perioperatively. A decreased score was maintained over 5 years in $46 \%$ of patients. A new adjacent vertebral fracture was documented by 33 vertebral bodies in 22 patients. During the follow-up period, 43 patients (38\%) in 112 patients died. Anterior body height in the last follow-up was improved about $0.3 \mathrm{~mm}$ compared with the preprocedural value, but was not statistically significant. Also, the focal kyphotic angle was reduced from $12.3^{\circ}$ at the preprocedural state to $11.7^{\circ}$ at the postprocedural state, but was not statistically significant $(p>0.05)$.

Conclusions: PVP for osteoporotic compression fracture is an efficient procedure for pain relief by long term follow-up. The cement injected vertebrae showed stable radiologic progression without significant changes in vertebral height or kyphotic angle.
\end{abstract}

Key Words: Percutaneous vertebroplasty, Long-term follow-up, Osteoporotic vertebral fracture

\section{Introduction}

Osteoporotic compression fracture is known to cause physical, functional and mental side-effects and complications such as lowered quality of life and increased mortality rate [1-3]. As a treatment procedure, vertebroplasty is widely used due to its non-invasiveness. Moreover, many studies reported improvements in clinical symptoms upon obtaining immediate postoperative pain relief and vertebral stability. According to the report of Jensen et al. [4] on vertebroplasty performed in 47 cases of osteoporotic compression fractures in 29 patients' vertebral bodies, $90 \%$ of the cases showed significant postoperative pain relief. In addition, Layton et al. [5] reported results of significant pain relief and functional improvement in 1,000 vertebral bodies from 552 patients. As explained, the efficacy of vertebroplasty is

Received May 25, 2011; Revised Aug 10, 2011; Accepted Aug 25, 2011

Corresponding author: Jung Hoon Kim, MD

Department of Orthopedic Surgery, Inje University Ilsan Paik Hospital, Inje University College of Medicine,

2240 Daehwa-dong, Ilsanseo-gu, Goyang 411-706, Korea

Tel: +82-31-910-7968, Fax: +82-31-910-7967, E-mail: oskim@paik.ac.kr 
well known, but the long-term clinical and radiologic results and the stability of injected cement are not well understood. The purpose of this study was to evaluate long-term clinical results and changes in the injected cement as well as the radiologic results analyzed over long-term follow-up.

\section{Materials and Methods}

\section{Materials}

We sampled patients who visited the hospital between January, 2000 and August, 2005 with the chief complaint of back pain due to compression fractures in thoracic or lumbar vertebrae caused by osteoporosis. Out of a total of 159 patients who underwent percutaneous vertebroplasty, 69 patients with 121 vertebrae cases were selected except 43 dead patients and 47 patients who were unavailable for follow-up for 5 years or more. Indications of the surgery were persistent pain after post-traumatic conservative treatments, or vertebral collapse upon progression of compression fracture. The 69 patients included 12 males and 57 females in the age range of 54-87 with a mean of 67 years old. A majority of patients were in their sixties and seventies, comprising 59 patients. In terms of the injured area, compression fractures in the 1st lumbar vertebra accounted for 31 cases, which was the highest amount, followed by 19 cases in the 12th thoracic vertebra, 17 cases in the 2nd lumbar vertebra, 14 cases in the 3rd lumbar vertebra, 13 cases in the 11th thoracic vertebra, 8 cases in the 4th lumbar vertebra, 6 cases in the 9th and 10th thoracic vertebrae, respectively, 5 cases in the 8th thoracic vertebra, and 1 case in the 6th and 7th thoracic vertebrae, respectively. The result of bone mineral densitometry showed a mean T-score of -3.3 .

\section{Preoperative patient evaluation, surgical technique and postoperative care}

Surgical indication of percutaneous vertebroplasty was performed for cases experiencing persistent pain or radiographicallly progressive collapse after conservative treatments followed by a reassessment after 3 weeks. Decision of symptomatic vertebrae was made on the areas that showed compression fractures through plain radiograph and focal tenderness through a physical examination, and hot uptake through a bone scan. In addition, degree and displacement of fracture fragments were checked through preoperative computed tomography (CT) and degree of os- teoporosis was measured using bone mineral densitometry. Additional magnetic resonance imaging (MRI) in some cases indicated that metastatic cancer was suspected based on medical history or plain radiologic results or time of developing bone fracture was unclear due to multiple compression fracture pathology. Cases of using MRI for other non-treatment reasons were also included.

Surgical technique includes: administration of fentanyl and midazolam which are premedications after preventive antibiotics, 30 minutes prior to surgery; local anesthesia on the surgical area with $1 \%$ lidocaine at prone position; pin point skin incision; insertion of 11-gauge J-type bone marrow biopsy needle into the lesion inside the vertebral body through both pedicles and confirmation of location of the needle under image intensifier. Bone cement (Surgical Simplex P, Howmedica, Limerick, Ireland) was mixed with tungsten powder, and injected into the vertebral body while monitoring cement leakage using a $\mathrm{C}$-arm image intensifier. The amount of cement loaded in the syringe was confirmed and recorded after the injection. Immediately after the surgery, activities on the bed and early ambulation were allowed, and a Juwett brace was provided for most of the thoracolumbar fracture cases.

\section{Methods}

In order to confirm objectified changes in pre- and postoperative pain levels, a visual analogue scale (VAS) with a scale of 0 (no pain) to 10 (most severe pain) was prepared to assess pain levels before the operation, and 2 days, 3 months, 1, 3 and 5 years after the operation. Anteoposterior and lateral radiographs were taken to measure anterior body height and kyphotic angle of vertebral bodies. Changes of injected cement were also retrospectively confirmed. The anterior body heights of collapsed vertebrae prior to compression was assumed as the mean $(a+b / 2)$ of the anterior body heights of the upper vertebral body (a) and the lower vertebral body (b) of the collapsed vertebra. Degree of compression was expressed as the ratio of anterior body height of the collapsed vertebrae (c) against the anterior body heights of collapsed vertebrae prior to compression. The kyphotic angle was measured as the angle $(\alpha)$ between the upper and lower end plate of the compressed vertebrae using the Cobb method (Fig. 1). Changes of cement injected into the symptomatic vertebrae and adjacent vertebrae were observed through follow-up X-ray. Upon comparing plain radiologic photographs in the progression observation pe- 
riod and immediately after surgery, changes in cement were classified as dissociation in the case of $2 \mathrm{~mm}$ or more of progressive radiolucent finding between the injected cement and trabecular bone of vertebral body, and as vertebral collapse in the case of $30 \%$ or more loss of vertebral height, and $20^{\circ}$ or more of changes in the focal kyphotic angle. Cracks on cement itself and between the vertebral body and cement were confirmed. In addition, invasion of cement into the end plate, and significant cement leakage to the posterior wall of the vertebra was also confirmed through plain radiographs.

During the progression observation period, presence and pattern of additional or new compression fractures occurring in the vertebral body, and the period between the surgery and the additional or new fracture were also investigated.

When deaths were confirmed, total mortality rate and 5-year mortality rate after vertebroplasty were analyzed. Deaths of patients transferred to other departments were confirmed with their medical records, and in the cases with no records available, interviews were conducted with the deceased patients' family over the phone. For statistical processing, paired $t$-test was used, and the significance level was set at $p<0.05$.

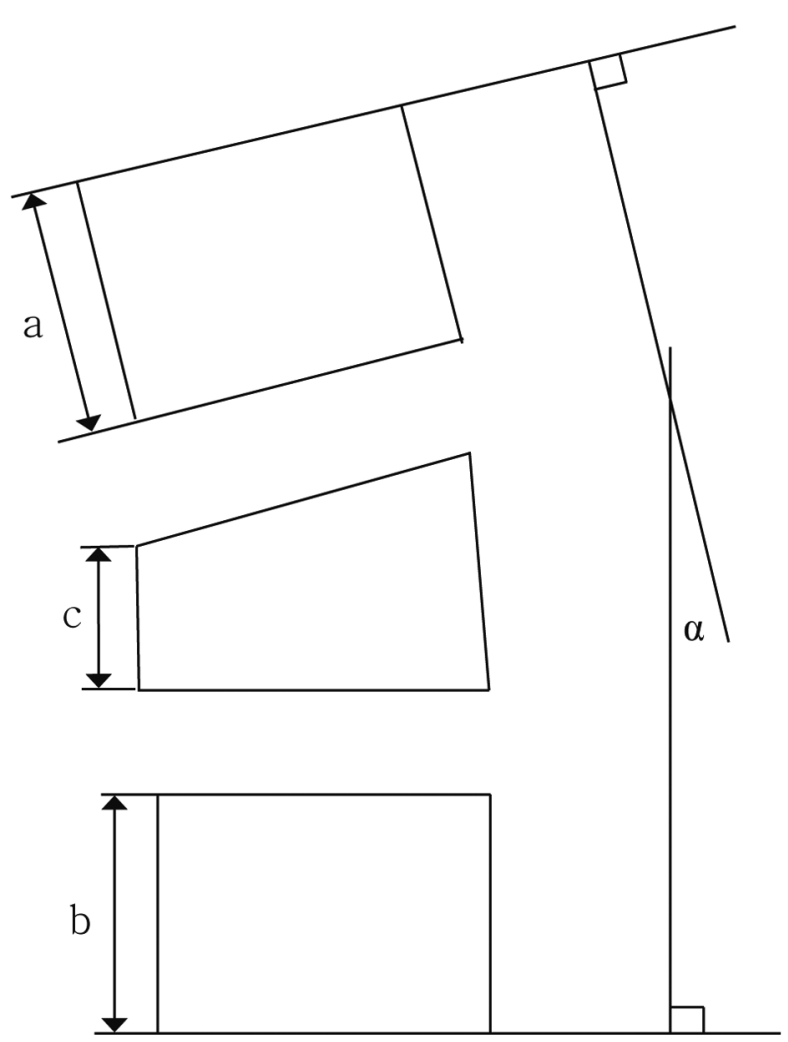

Fig. 1. Measurement methods for anterior vertebral height and kyphotic angle $(\alpha)$.

\section{Results}

\section{Clinical results}

The average follow-up period was 5.7 years with a minimum of 5 years and a maximum of 9.5 years. Pain levels according to the VAS reduced by a value of 4.9 from the preoperative mean of 7 to the perioperative mean of 2.1. A total of $46 \%$ of the patients reported a 5 or more for reduction and maintenance of their pain level (Fig. 2).

During the follow-up period, 33 cases of newly developed vertebral fracture were reported in the vertebral bodies of 22 patients which signified $32 \%$ newly developed vertebral fractures. The 33 cases comprised of 11 cases of adjacent vertebral fracture and 22 cases of non-adjacent vertebral fracture, whereas 20 patients with 31 vertebral bodies underwent additional percutaneous vertebroplasty, and 2 patients had conservative treatments.

The number of patients that died during the follow-up period reached 43 out of 112 patients that underwent vertebroplasty excluding 47 patients who were not able to be monitored. Out of 43 patients that died, 24 patients died within 5 years representing $56 \%$ of the total. With regards to the time of death, 4 patients died within 1 year of surgery $(10 \%), 8$ died between 1 and 2 years (19\%), and 5 died between 2 and 3 years $(12 \%)$. The mean age of the deceased patients was 76.9 years old.

\section{Radiologic results}

The mean anterior vertebral heights were $17.45 \mathrm{~mm}$ before surgery, $20.8 \mathrm{~mm}$ immediately after surgery, and 17.7 $\mathrm{mm}$ at the end of 5-year follow-up, which signified a 0.3

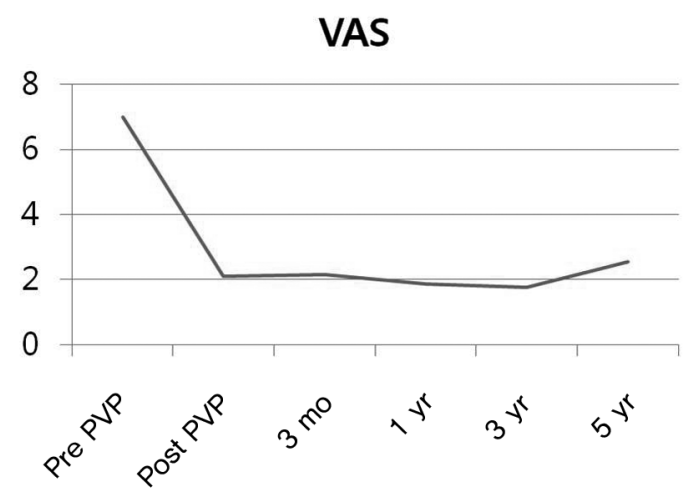

Fig. 2. Visual analogue scale (VAS) score was decreased and then maintained after percutaneous vertebroplasty (PVP). 
Table 1. Anterior vertebral height and kyphotic angle

\begin{tabular}{lccccccc}
\hline \hline & Pre OP & Post OP & 3 mo & 1 yr & 3 yr & 5 yr & $p$-value \\
\hline Anteriorvertebral heights $(\mathrm{mm})$ & 17.45 & 20.8 & 19.42 & 18.97 & 19.52 & 17.70 & $>0.05$ \\
Kyphotic angle $\left(^{\circ}\right)$ & 12.3 & 9.63 & 10.86 & 9.67 & 10.76 & 11.67 & $>0.05$ \\
\hline
\end{tabular}

OP: Operative.

$\mathrm{mm}$ increase that was not statistically significant $(p>0.05)$. The kyphotic angles were $12.30^{\circ}$ before surgery, $9.63^{\circ}$ after surgery, and $11.67^{\circ}$ at the end of the 5-year followup, showing a $0.6^{\circ}$ reduction from the preoperative angle, but these findings ere not statistically significant $(p>0.05)$ (Table 1).

The mean amount of injected cement was $5.2 \mathrm{ml}$. Out of the 69 cases, the 63 cases for whom the cement was injected into the vertebral body were kept in a stable condition. Two cases of radiolucent line with decreased bone density in the adjacent area of cement, and accompanying dissociation were observed (Fig. 3), and 4 cases of cement cracks accompanied with vertebral collapse were observed (Fig. 4). The 2 dissociation cases did not show clinically significant findings such as pain, and no additional procedure was performed. Three out of 4 cases in which the cement cracks accompanied vertebral collapse developed collapse 3 years after successful operation, and 1 case developed collapse in 8 months. The latter case had a deformity of more than $24^{\circ}$ of preoperative focal kyphotic angle, and the deformity was not corrected by surgery and a persistent collapse developed despite some symptomatic improvements. In all 4 cases, collapse-related pain did not develop, and no additional fixation or repeated operation was performed. In 1 case of
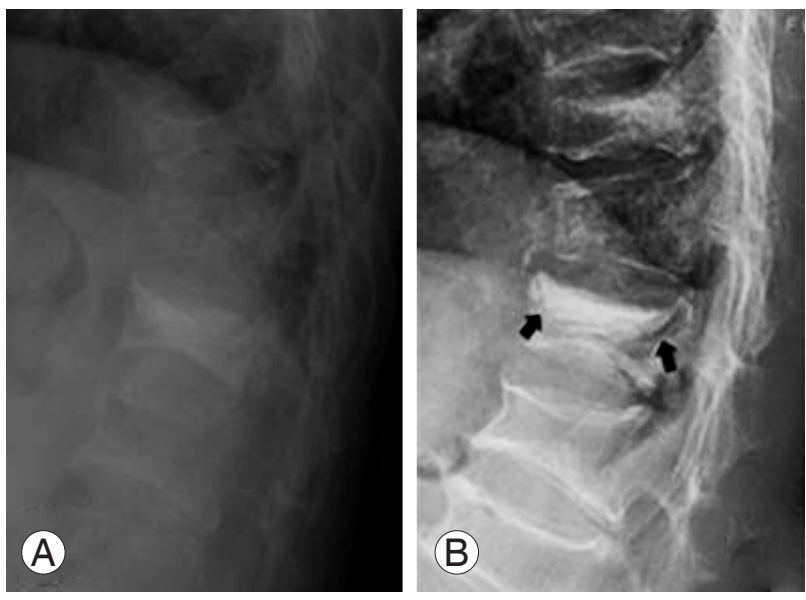

Fig. 3. Postoperative radiographs of a 71-year-old woman with an osteoporotic compression fracture on T12. (A) Immediately postoperative radiograph showed percutaneous vertebroplasty on T12. (B) Postoperative 8 years follow-up radiograph showed radiolucentic area between cement and bone. dissociation, radiolucent changes between cement and the upper end plate, and cement and posterior wall of vertebra were found between 1 and 3 years after surgery. In another case, radiolucent changes between cement and the lower end plate were observed 5 years after surgery. In both cases, no changes in kyphotic angle or vertebral height, and no associated symptoms were observed. Regarding plain radiographic changes in the adjacent vertebrae, 10 out of 69 cases showed findings of spontaneous fusion from bony bridge in the 3 sides or more cortex of the proximal vertebra, and progressive fusion was found in 4 cases. Overall, $20 \%$ of the total cases showed changes between cement injected vertebrae and the adjacent vertebrae.

\section{Complications}

During the follow-up, cement leakage out of the end plate was confirmed in 29 cases out of 121 vertebral bodies which were available of progression observation, but no symptoms developed in all 29 cases and therefore no additional treatments were provided. In all 4 cases with cement cracks and collapse in the vertebral body showed leakage out of the upper end plate. The mean amount of injected cement was $5.9 \mathrm{ml}$, which exceeds the mean injection amount,
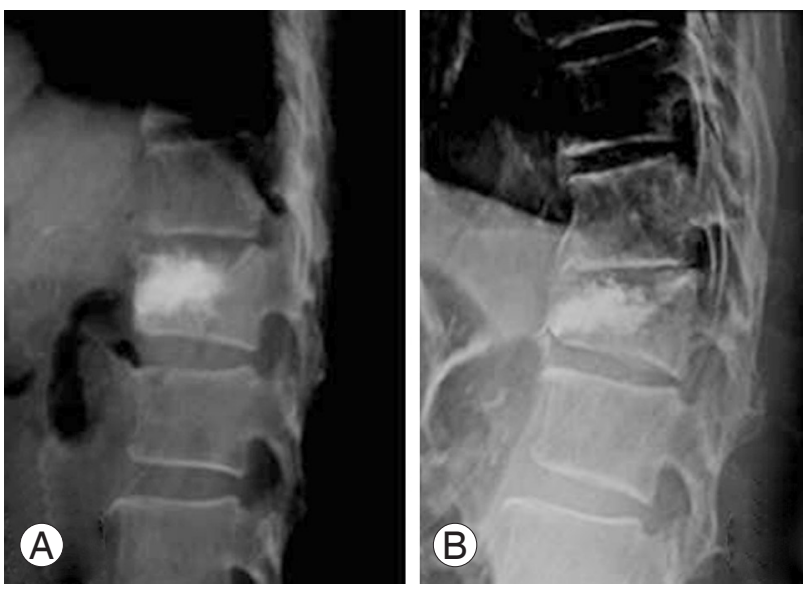

Fig. 4. Vertebral collapse with cement destruction in a 75-year-old man. (A) Immediately postoperative radiograph of L1. (B) Postoperative 5.5 years follow-up radiograph showed a mild vertebral collapse with increased focal kyphotic angle. 
but statistical significance could not be determined due to a lack of data. Also, 2 cases developed neurologic symptoms due to cement leakage into the spinal canal. In one of these, laminectomy and decompression were performed using
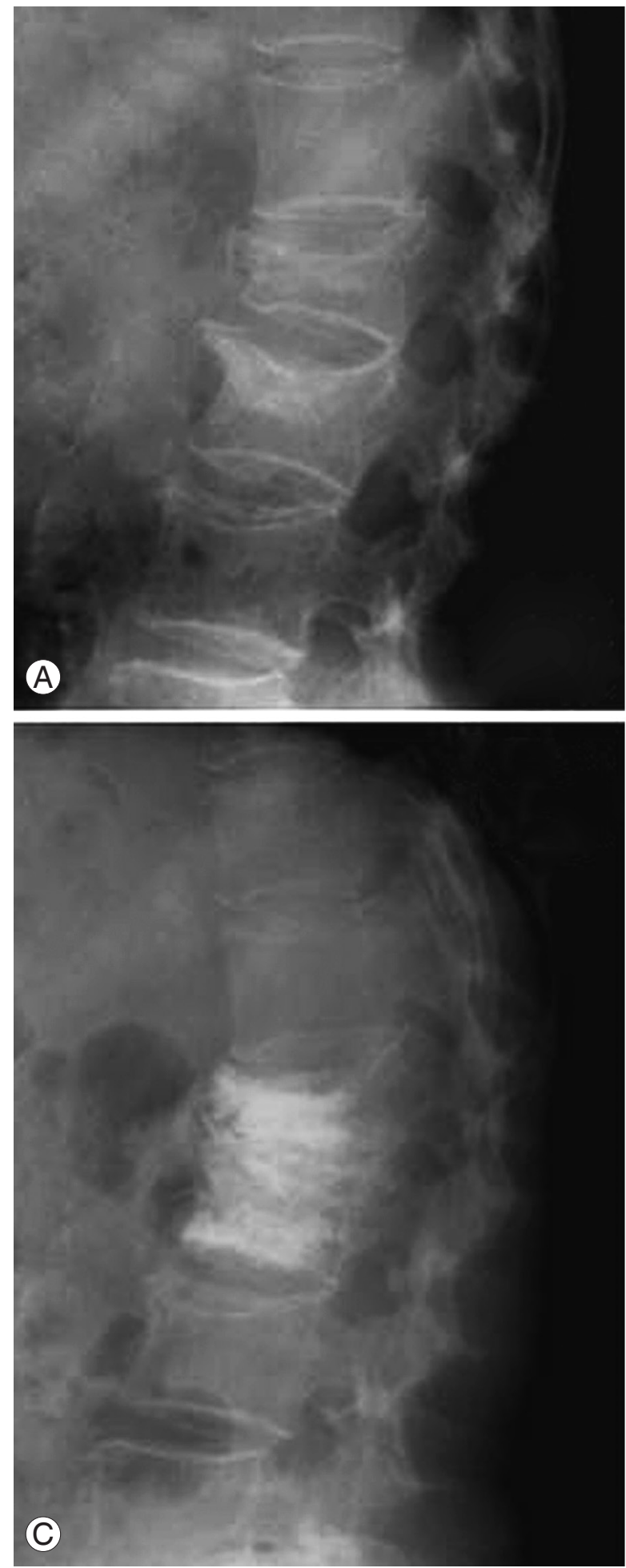

\section{B}

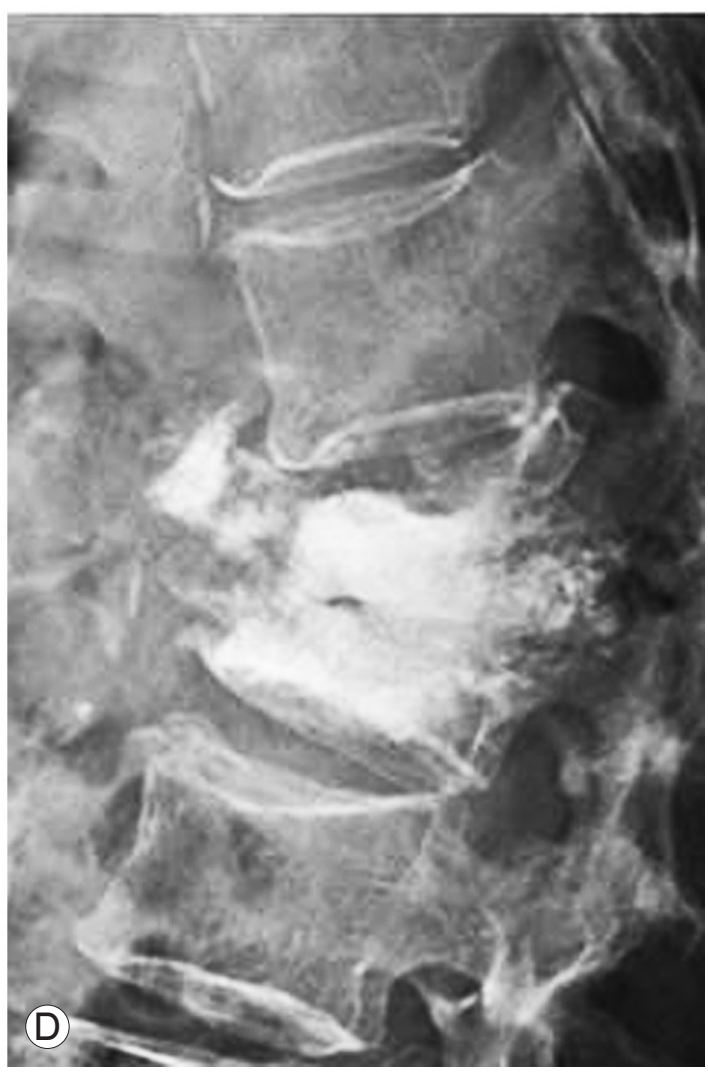

Fig. 5. Simple lateral radiographs of a 70-year-old woman with L1,2 compression fracture. (A) Preoperative radiograph showed L1,2 compression fracture. (B) Postoperative radiograph showed percutaneous vertebroplasty on L1,2. (C) Postoperative 2 years follow-up radiograph showed a increased focal kyphotic angle between L1 and L2. (D) Postoperative 6 years follow-up radiograph showed a further increased kyphotic angle between L1 and L2. 
vertebroplasty due to misdiagnosing preoperative tuberculous spondylitis to pathologic fracture, but the case was also excluded from the study as the patient dropped out of the progression observation.

1) Case 1: a collapse example of cement and vertebral body during the follow-up

A 70-year-old female patient received conservative treatment due to an osteoporotic compression fracture in the 1st and 2nd lumbar vertebrae, but showed no improvement in symptoms. Then, she underwent percutaneous vertebroplasty, and the pain reduced immediately after the surgery (VAS score, 9 to 4). One year after the surgery, however, a gradual increase in focal kyphotic angle and collapse around the cement were observed, and the trend continued until the 6th year of follow-up (Fig. 5). According to CT and MRI findings (Fig. 6), protrusion of a bony fragment into the spinal canal was confirmed, but neurologic symptoms or aggravation in pain have not been observed yet, and the case is still under observation.

2) Case 2: an example of cement which remained stable during the follow-up based on plain radiologic results

A 67-year-old female patient received conservative treatment due to osteoporotic compression fractures of the 10th and 12th thoracic vertebrae which occurred when she fell, but she did not experience any improvement in symptoms. After undergoing percutaneous vertebroplasty, VAS score reduced from 6 to 3, and after 8 years of follow-up, VAS score of 0 and stable vertebrae were observed on plain radi-
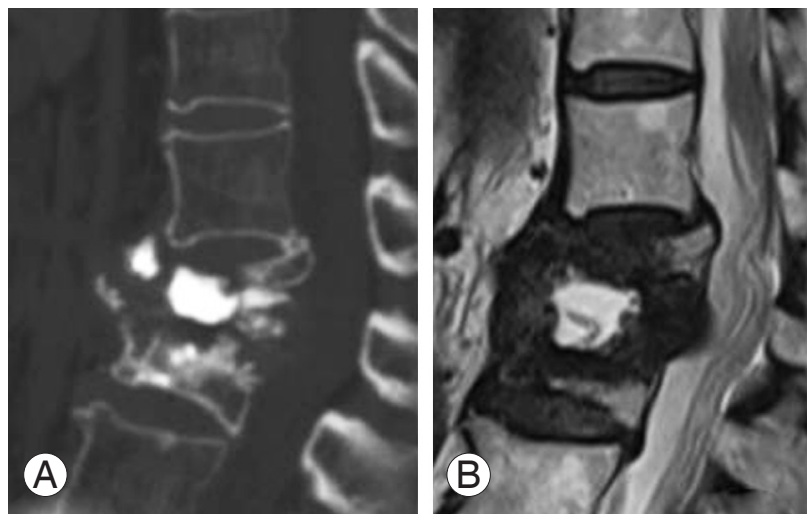

Fig. 6. Computered tomography (CT) and magnetic resonance image (MRI) of a 70-year-old woman with L1,2 compression fracture. (A) Postoperative 6 years follow-up CT image shows destructed bony structure and cement. (B) Postoperative 6 years follow-up MRI shows a protrusion of destructed cement to spinal canal. ography (Fig. 7).

3) Case 3: a case of spontaneous fusion with adjacent vertebra

A 73-year-old female patient underwent percutaneous vertebroplasty due to an osteoporotic compression fracture of the 1st lumbar vertebra, which occurred when she fell. In the 3rd year of progression observation, the patient experienced another injury with a newly developed fracture of the 12 th thoracic vertebra. Following that, findings of spontaneous fusion among vertebral segments were reported (Fig. 8).

\section{Discussion}

The purpose of the present study was to confirm the clinical and radiologic results of 69 patients who underwent vertebroplasty and were available for 5 years or more of follow-up. The patients were confirmed with radiologic results including related symptoms and cement changes. Currently, percutaneous vertebroplasty is performed for cases of osteoporotic compression fracture that do not respond to conservative treatments. Many studies have been conducted on clinical efficacy. According to Jansen et al. [4], 90\% of patients reported symptom improvements, and according to a study by Barr et al. [6], 63\% of patients reported symptom improvements after receiving percutaneous vertebroplasty. In the present study, symptom improvements were shown with the reduced VAS value by 5 marks on a short-term basis. Likewise, satisfactory reductions in clinical symptoms were reported through the long-term studies including 1 year or more of follow-up on a group of 25 patients con-
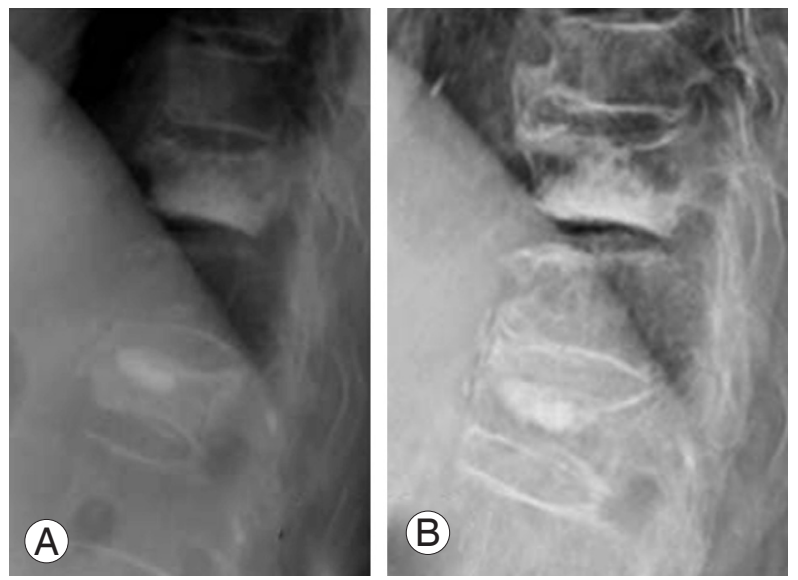

Fig. 7. Simple lateral radiographs of a 67-year-old woman with T10 and T12 compression fracture. (A) Immediately postoperative radiograph of T12. (B) Postoperative 8 years follow-up radiograph show no interval change. 
ducted by Grados et al. [7], and 5 years or more of followup on a group of 13 patients conducted by Pérez-Higueras et al. [8]. We also confirmed the effectiveness of the procedure with reduced and then maintained VAS value by 5 marks or more in $46 \%$ of the total cases. As explained, percutaneous vertebroplasty is considered to be continuously effective for reducing pain caused by compression fracture on both a short and long-term basis.

As the present study was conducted with elderly patients, many deaths were confirmed during progression observation. Out of 159 patients, 112 were available for progression observation, and 43 patients (38\%) died during this period.
According to other studies, Grados et al. [7] reported 10 deaths $(29 \%)$ out of 35 patients which were available for follow-up. High mortality rate is caused by a high incidence of fractures in elderly people, but on the other hand, the reason is that bone fractures in old age may lead to an increase in mortality rate [9]. Vertebral compression fracture may also be a factor that can increase the mortality rate, but further study about this is necessary.

During the study period, 33 cases of new fracture including 11 adjacent fractures in the vertebral body in 22 out of 69 patients occurred. Through percutaneous vertebroplasty, cement is injected into the vertebral body in a minimally
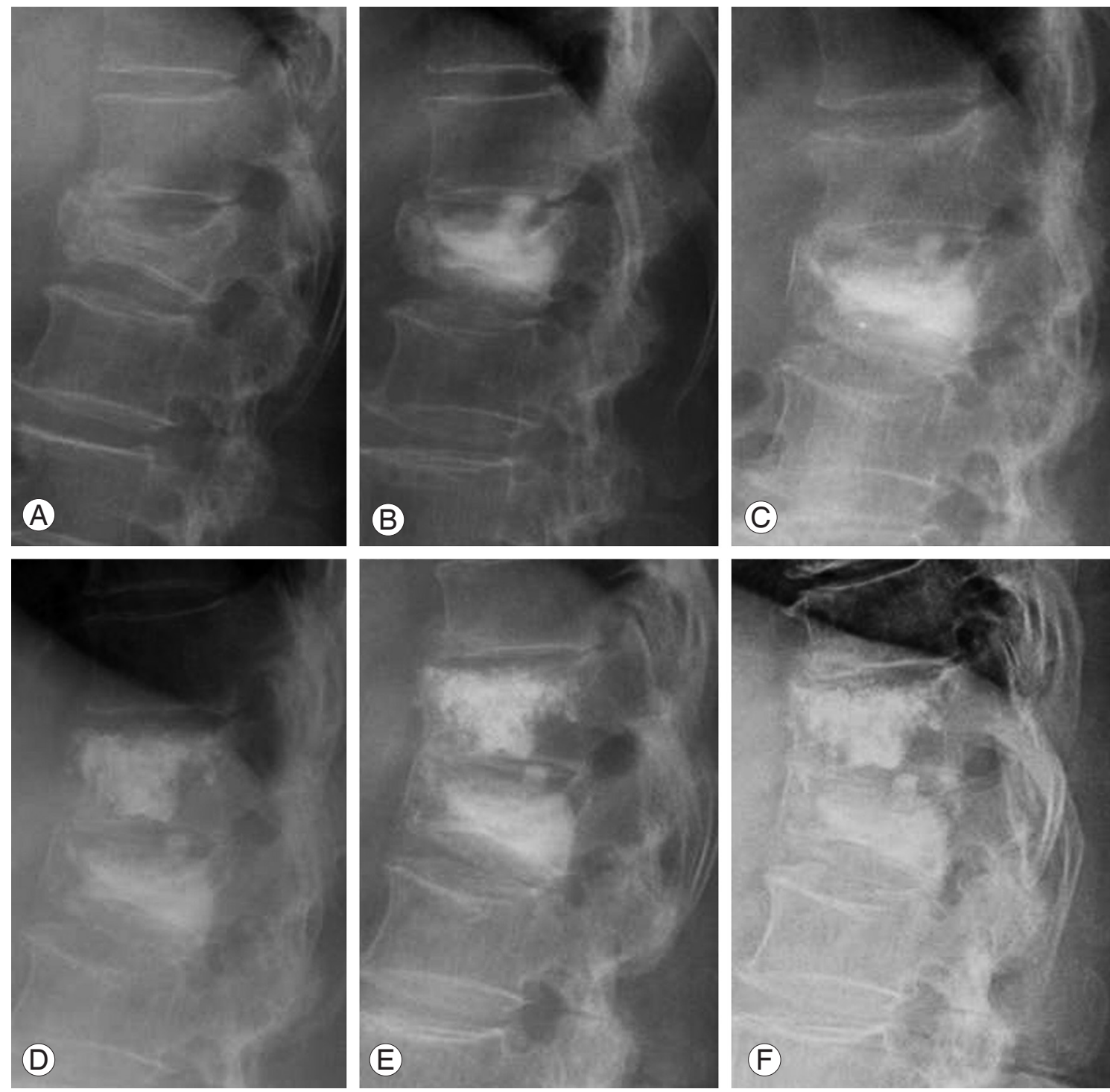

Fig. 8. Simple lateral radiographs of a 73-year-old woman with L1,T12 compression fracture. (A) Preoperative radiograph showed L1 compression fracture. (B) Immediately postoperative radiograph of L1. (C) Postoperative 3 years follow-up radiograph show a new compression fracture on T12. (D) Immediately postoperative radiograph of T12 (3 years after L1 postoperatively). (E) Two years after vertebroplasty on T12, follow-up radiograph show a progressive bony bridging between T12 and L1. (F) Four years after vertebroplasty on T12, follow-up radiograph show a spontaneous fusion between T12 and L1. 
invasive way to provide the fractured vertebra with strength. The widely used poly-methyl methacrylate (PMMA) offers $100 \mathrm{MPa}$ compressive strength which is higher than the trabecullar bone ( $3 \mathrm{MPa}$ ), and lower than the cortical bone (175 $\mathrm{MPa}$ [10]. Accordingly, fractures in the adjacent vertebrae may occur due to the difference in strength from the surrounding vertebrae. Uppin et al. [11] reported that the new bone fractures rate was $12.4 \%$ and $67 \%$ of these occurred in the adjacent bones.

As far as radiologic changes in vertebral height or local kyphotic angle after vertebroplasty are concerned, various views have been presented, and this has been known as one of the theoretical backgrounds of balloon kyphoplasty in the biomechanical perspective. According to the present study, the vertebral height or focal kyphotic angle improved right after surgery were not maintained long-term follow-up.

Changes of injected cement and vertebral body were partially investigated in several cases, but no long-term investigation using plain radiologic results was attempted to date. Togawa et al. [12] reported that histologically there is no direct bony union between the cement and bone but a thin fibrous membrane is filled. Braunstein et al. [13] confirmed a large amount of woven bone around the injected cement.

In the present study, long-term changes of cement injected into the body are evaluated using radiologic results. According to the present study, cement itself did not change but symptoms of dissociation, crack and vertebral collapse developed as complications occurring from the interaction between the vertebral body and cement. Many studies on short-term complications of vertebroplasty have been conducted, and the incidence rate of complications is known to be low $[4,6]$. PMMA is a comparatively stable cement and has been widely used. According to the 5-year radiologic results reported by Pérez-Higueras et al. [8], there were no cases of dislodgment, cracks or vertebral collapse in the 13 cases of injected cement. Tsai et al. [14] reported a rare case of anterior dislodgment accompanied with neurologic symptoms in the bone cement injected after surgery that required additional surgery. In the present study, 2 cases of dissociation around the cement, and 4 cases of vertebral collapse accompanied with cement cracks were confirmed. The 2 cases of dissociation showed a linear change which can be considered an osteolytic lesion without a change in clinical symptoms. Since the change does not progress upon occurrence, and no collapse or accompanying transformation in the angle is seen, the change is considered clinically not significant. All 4 cases of collapse showed postoperative leakage without accompanying symptoms. In detail, the first case of vertebroplasty at the two neighboring vertebrae reported an increase in kyphotic angle, gradually increasing vertebral compression and cement collapse at the surrounding area of lower segment 1 year after the surgery. Similar to the case reported by Tsai et al. [14], defection at the anterior cortical bone of the lower segment is considered to affect the collapse. The second and third cases reported an increase in the preoperative kyphotic angle by $20^{\circ}$ or more, and a decrease in vertebral body height by $70 \%$. The postoperative kyphotic angle did not recover but collapsed later. The last case with a preoperative defect in the anterior cortical bone reported a degenerative spondylopathy 2 years after the surgery, which resulted in a narrowing anterior intervertebral disc space initially and then, minimal cement collapse as well as changes in the kyphotic angle. Increase in the kyphotic angle contributes to increase in the compression force at the injected cement, the corresponding vertebral body and surrounding vertebral bodies. Consequently, fractures in the neighboring vertebral bodies may happen, and in a few cases, collapse of the vertebral segment which is the site of surgery may also occur. More specifically, if there is a significant increase in preoperative kyphotic angle by $20^{\circ}$ or more, a decrease in vertebral body height, defect or destruction of anterior cortical bone, or cement leakage in association with the surgery, cement cracks or vertebral collapse may appear. As mentioned above, even though the bone cement used for vertebroplasty immediately relieves peri-operative pain, and enhances loading capacity to stabilize vertebral body, it does not assimilate during metabolism in the vertebral bodies and still remains as a foreign material which may result in a lower biocompatibility and a problem of bone absorption between the bone and the cement [15]. In an attempt to overcome the drawbacks of bone cement, vertebroplasty using bone replacements such as hydroxyapatite, hydroxyapatite/tricalcium phosphate and coral bone have been suggested [16,17], but extensive studies on verifying the comparative advantage of these materials against bone cement will be necessary. In the 5 years or more of progression observation on 121 vertebral bodies of 69 patients in the present study, most of the bodies were confirmed to be in a stable condition but 6 vertebral bodies (about 5\%) showed dissociation, crack or collapse in association with the site of surgery requiring meticulous observations and attention before and during operations. The spontaneous fusion findings obtained during the follow-up are assumed to be a result of the role that the bone cement played on biomechanical stability, at least partially. Despite stable progress of the cement-injected vertebrae on a long- 
term basis confirmed by the present study, contradictory results inferring biomechanical effects to some extent were also obtained. Accordingly, biomechanically stable materials having high biocompatibility will be necessary in order to prevent the dissociation between the cement, bone, and vertebral collapse.

This study had a number of limitations. Firstly, it is a retrospective study without a control group, and comparisons with conservative treatments and other treatment modalities were not conducted. In addition, the number of dropouts during the follow-up was 47 , comprising $30 \%$ of the total of 159 , which is considerably high. Moreover, analysis on the causes and risk factors of patient death were not conducted. Considering that the subjects were elderly patients, further studies on deaths and dropouts are necessary in the future.

\section{Conclusions}

PVP for osteoporotic compression fracture is an efficient procedure for pain relief by long-term follow-up. However, this procedure has also shown a comparatively high new fracture rate and mortality rate. The cement injected vertebrae showed stable radiologic progression without significant changes in vertebral height or kyphotic angle.

\section{Acknowledgements}

This work was supported by grant from Inje University, 2008.

\section{REFERENCES}

1. Jung HW, Park JY, Kim KJ, Lee JC, Kim YI, Shin BJ. Conservative treatment of compression and stable burst fractures in the thoracolumbar junction: early ambulation vs. late ambulation. J Korean Orthop Assoc 2002;37:483-8.

2. Suk SI, Lee CK, Kang HS, et al. Vertebral fracture in osteoporosis. J Korean Orthop Assoc 1993;28:980-7.

3. Weinstein JN, Collalto P, Lehmann TR. Thoracolumbar "burst" fractures treated conservatively: a long-term follow-up. Spine (Phila Pa 1976) 1988;13:33-8.

4. Jensen ME, Evans AJ, Mathis JM, Kallmes DF, Cloft HJ, Dion JE. Percutaneous polymethylmethacrylate vertebroplasty in the treatment of osteoporotic vertebral body compression fractures: technical aspects. AJNR Am J Neuroradiol 1997;18:1897-904.

5. Layton KF, Thielen KR, Koch CA, et al. Vertebroplas- ty, first 1000 levels of a single center: evaluation of the outcomes and complications. AJNR Am J Neuroradiol 2007;28:683-9.

6. Barr JD, Barr MS, Lemley TJ, McCann RM. Percutaneous vertebroplasty for pain relief and spinal stabilization. Spine (Phila Pa 1976) 2000;25:923-8.

7. Grados F, Depriester C, Cayrolle G, Hardy N, Deramond $\mathrm{H}$, Fardellone P. Long-term observations of vertebral osteoporotic fractures treated by percutaneous vertebroplasty. Rheumatology (Oxford) 2000;39:14104.

8. Pérez-Higueras A, Alvarez L, Rossi RE, Quiñones D, Al-Assir I. Percutaneous vertebroplasty: long-term clinical and radiological outcome. Neuroradiology 2002;44:950-4.

9. Lau E, Ong K, Kurtz S, Schmier J, Edidin A. Mortality following the diagnosis of a vertebral compression fracture in the medicare population. J Bone Joint Surg Am 2008;90:1479-86.

10. Rockwood CA, Green DP, Bucholz RW. Rockwood and Green's fractures in adults. Vol. 3. 6th ed. Philadelphia, PA: Lippincott Williams \& Wilkins; 2006. p. 4-11.

11. Uppin AA, Hirsch JA, Centenera LV, Pfiefer BA, Pazianos AG, Choi IS. Occurrence of new vertebral body fracture after percutaneous vertebroplasty in patients with osteoporosis. Radiology 2003;226:119-24.

12. Togawa D, Bauer TW, Lieberman IH, Takikawa S. Histologic evaluation of human vertebral bodies after vertebral augmentation with polymethyl methacrylate. Spine (Phila Pa 1976) 2003;28:1521-7.

13. Braunstein V, Sprecher CM, Gisep A, et al. Long-term reaction to bone cement in osteoporotic bone: new bone formation in vertebral bodies after vertebroplasty. J Anat 2008;212:697-701.

14. Tsai TT, Chen WJ, Lai PL, et al. Polymethylmethacrylate cement dislodgment following percutaneous vertebroplasty: a case report. Spine (Phila Pa 1976) 2003;28:E457-60.

15. Cunin G, Boissonnet H, Petite H, Blanchat C, Guillemin G. Experimental vertebroplasty using osteoconductive granular material. Spine (Phila Pa 1976) 2000;25:1070-6.

16. Bostrom MP, Lane JM. Future directions: augmentation of osteoporotic vertebral bodies. Spine (Phila Pa 1976) 1997;22(24 Suppl):38S-42S.

17. Cotten A, Duquesnoy B. Vertebroplasty: current data and future potential. Rev Rhum Engl Ed 1997;64:6459. 